\title{
Interleukin 8 enhances the immune response of ducks to avian influenza vaccine
}

\author{
Y. RUAN ${ }^{1}$, X. JI ${ }^{1,2, *}, M . W^{2} N^{1,2}, X . Z^{2} U^{1}, X . F^{1}$ \\ ${ }^{1}$ College of Animal Science, Guizhou University, Guiyang, 550025, P. R. China; ${ }^{2}$ Laboratory for Animal Disease of Guizhou Province, \\ Guizhou University, Guiyang, 550025, P. R.China
}

Received January 7, 2014; accepted November 3, 2014

\begin{abstract}
Summary. - Interleukins are reported to be valuable immunostimulants in enhancing the immune efficiency of conventional vaccines. In this study, the effect of expression of interleukin 8 (IL-8) on the immune response of ducks to avian influenza vaccine was investigated. The results showed that the serum antibody titer, lymphocyte transformation efficiency and serum interferon gamma (IFN- $\gamma$ ) level of ducks injected with avian influenza vaccine along with a plasmid expressing duck IL-8 were higher than those of ducks injected with conventional immunostimulant Astragalus polysaccharide (APS) or empty plasmid. Therefore, the duck IL- 8 may be used as a good immunostimulant to enhance the immune efficiency of avian influenza vaccine in ducks.
\end{abstract}

Keywords: interleukin 8; avian influenza virus; vaccine; Astragalus polasaccharide; duck; immune response

\section{Introduction}

In recent years, highly pathogenic avian influenza $\mathrm{H} 5$ virus has caused considerable losses to poultry industry around the world and has caused a great threat to public health in several countries since the first appearance in 1996 (Capua and Alexander, 2007; Giese et al., 2008; He et al., 2013). Many studies revealed that domestic ducks are one of the primary natural reservoirs of avian influenza viruses (AIVs) of different subtypes, and they have been always thought to be the interface between the natural gene pool of wild aquatic birds and land-based poultry in the ecology of influenza viruses (Webster et al., 1992; Ellis et al., 2004). The conventional animal production pattern makes it possible for domestic ducks to contact with wild waterfowls and terrestrial poultry simultaneously, providing the opportunities to transmit viruses asymptomatically from the former to

*Corresponding author. E-mail: jxq972@aliyun.com; phone: +8613608560972.

Abbreviations: APS = Astragalus polysaccharide; $\operatorname{AIV}(\mathrm{s})=$ avian influenza virus(es); IFN- $\gamma=$ interferon gamma; IL-8 = interleukin 8; p.i. $=$ post immunization the latter. Therefore, it is important to prevent the potential transmission of AIVs from ducks to other susceptible animals or humans.

Several studies have suggested that the immune efficiency of AIV vaccines in ducks was lower than that in chickens (Webster and Hulse, 2005). To improve the immune response of AIV vaccines in ducks, immunostimulants were applied to enhance the production of IFNs and regulate immune function in immunized ducks (Yao et al., 2010; Garçon et al., 2012). In this study, we evaluated duck IL-8 and Astragalus polysaccharide (APS) as immunostimulants in enhancing the immune response of ducks to an AIV vaccine.

\section{Materials and Methods}

Ducks and vaccine. Five-day-old ducks (clean animals) were purchased from Sansui Duck Breeding Farm, Sansui, Guizhou, China. AIV vaccine (Recombinant avian influenza inactivated vaccine (H5N1 subtype, Re-6 strain) was purchased from Qingdao Yibang Biological Engineering Co., Qingdao, China.

Plasmid construction. The ORF of duck IL-8 gene (GenBank Acc. No. AB236335) was synthesized and inserted into eukaryotic 
expression plasmid pcDNA3.1 (+) (Invitrogen, USA) at the XhoI and BamHI sites to generate pcDNA3.1-dIL-8. The obtained clones were verified by DNA sequencing.

Animal experiment. Ducks were injected with the AIV vaccine and then randomly divided into three experimental groups (IL-8, empty plasmid and APS). Ten and 24 days post immunization (p.i.) with $0.5 \mathrm{ml}$ of avian influenza vaccine, the IL- 8 and the empty plasmid groups were injected with $200 \mu \mathrm{g}$ pcDNA3.1-dIL-8 and pcDNA3.1, respectively, whereas the APS group was injected with $0.01 \mathrm{~g}$ APS. The schedules and doses of vaccination as well as injection of immunostimulants were performed according to the instructions of AIV vaccine manufacturer: the first immunization and the second immunization were performed at 10- and 24-day-old, respectively, with the injection dose of avian influenza vaccine $0.5 \mathrm{ml}$ per animal. The schedules of immunostimulant injection were carried out as described previously (Yu et al., 2012). Unvaccinated and plasmid-uninjected ducks were used as blank control.

The weight of ducks was recorded and the blood was collected from the wing vein on days 10, 24, 38, 52, and 66 p.i. Sera were used for determination of lymphocyte transformation efficiency by MTT assay (Wu et al., 2007), serum antibody titer by hemagglutinationinhibition test and serum IFN- $\gamma$ level by ELISA as previously described (Yun et al., 2000).

\section{Results and Discussion}

In this study, the effect of expression of duck IL-8 in ducks and injection of conventional immunostimulant APS on the immune response of ducks to an AIV vaccine was investigated. The results showed that the antibody titer was undetectable in all vaccinated groups on day 10, but increased from day 24 to day 52 p.i. (Fig. 1a). The highest antibody titer levels were exhibited by the IL- 8 group, followed by the APS group and the empty plasmid group. The corresponding differences on days 38, 52 and 66 were significant $(\mathrm{P}<0.01)$.

As for the Tlymphocyte transformation efficiency, it was very low on day 10 in all the four groups, but then it increased continuously on days 24 and 38 to maximum values, which were reached in the IL- 8 group, whereas the APS group showed significantly lower values $(\mathrm{P}<0.01)$ (Fig. 1b). (a)

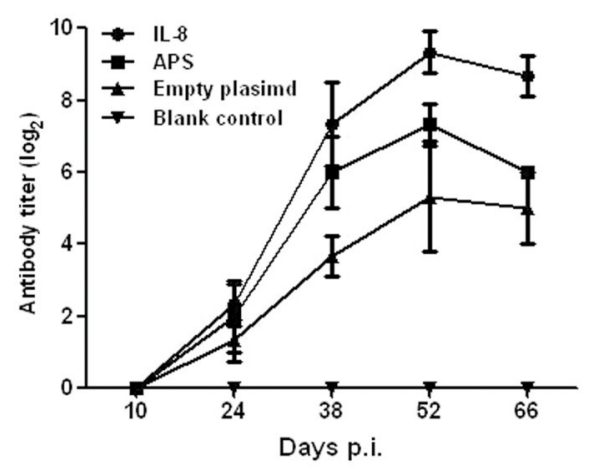

(c)

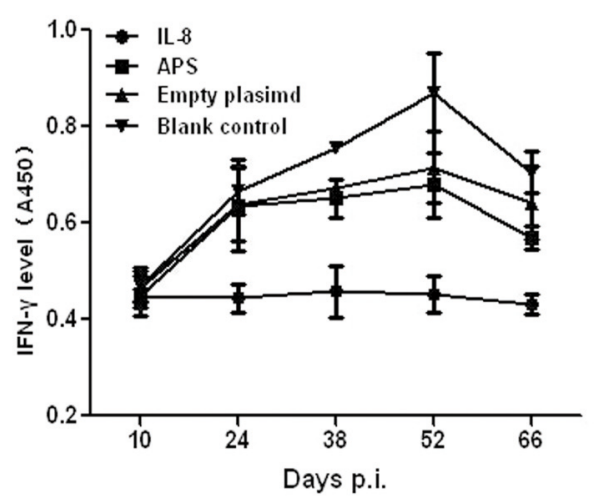

(b)

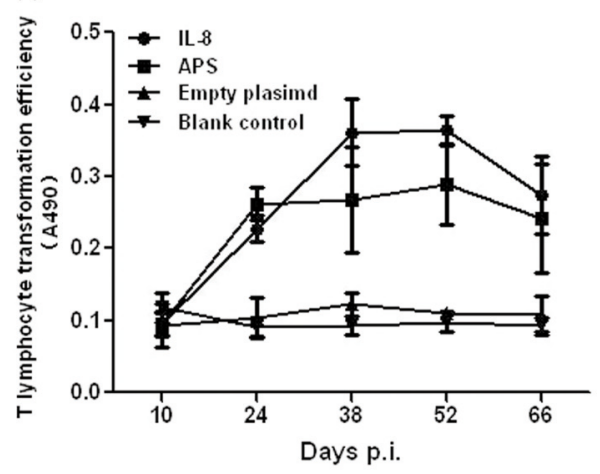

(d)

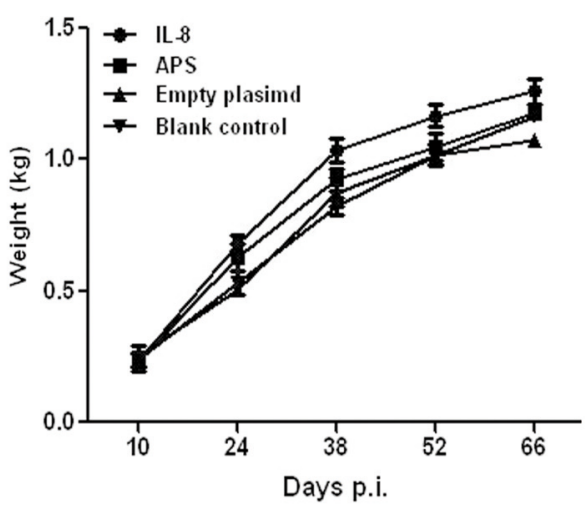

Fig. 1

Effects of IL-8 on the immune response of ducks to AIV vaccine

The serum antibody titer $\left(\log _{2}\right)(a)$, lymphocyte transformation efficiency $\left(\mathrm{A}_{490}\right)(\mathrm{b})$, serum IFN- $\gamma$ level $\left(\mathrm{A}_{450}\right)(\mathrm{c})$, and weight of ducks on days 10-66 p.i. $(\mathrm{kg})(\mathrm{d})$ in duck IL-8 group, APS group, empty plasmid group and blank control group were investigated. 
Assays of serum IFN- $\gamma$ levels showed that they increased continuously from day 10 to 52 in all three vaccinated groups to levels descending in the order IL-8, APS and empty plasmid group (Fig. 1c).

Eventually, the recording of weight of ducks showed a picture similar to those from previous assays, namely the highest weight increase in the IL-8 group, followed closely by the APS and the empty plasmid group (Fig. 1d), indicating that the use of IL-8 did not affect the weight growth of ducks.

Taken together, our study demonstrated that the effect of duck IL- 8 on the immune response of ducks to AIV vaccine was superior to that of conventional immunostimulant APS, providing a candidate immunostimulant in enhancing the immune efficiency of AIV vaccine in ducks.

Acknowledgements. The authors thank Dr. Zhiqiang Duan for his valuable advice and review of the manuscript. This work was supported by the grants from the Major Science and Technology Projects in Guizhou Province (No. QKHZDZX 2012-6004), the Science and Technology Plan in Guizhou Province (No. QKHJSH 2013-7004), and the Chinese Nature Science Foundation (No. 31260607).

\section{References}

Capua I, Alexander D (2007): Animal and human health implications of avian influenza infections. Bioscience Rep. 27, 359-372. http://dx.doi.org/10.1007/s10540-007-9057-9

Ellis TM, Bousfield RB, Bissett LA, Dyrting KC, Luk GSM, Tsim ST, Sturm-ramirez K, Webster RG, Guan Y, Peiris JSM (2004): Investigation of outbreaks of highly pathogenic $\mathrm{H} 5 \mathrm{~N} 1$ avian influenza in waterfowl and wild birds in Hong Kong in late 2002. Avian. Pathol. 33, 492-505. http://dx.doi.org/10.1080/03079450400003601

Garçon N, Stanley H, Martin F (2012): Evolution of adjuvants across. Vaccines, Expert Consult-Online. 58.

Giese M, Harder TC, Teifke JP, Klopfleisch R, Breithaupt A, Mettenleiter TC, Vahlenkamp TW (2008): Experimental infection and natural contact exposure of dogs with avian influenza virus (H5N1). Emerg. Infect. Dis. 14, 308. http:// dx.doi.org/10.3201/eid1402.070864

He L, Zhao G, Zhong L, Liu QT, Duan ZQ, Gu M, Wang XQ, Liu XW, Liu XF (2013): Isolation and characterization of two $\mathrm{H} 5 \mathrm{~N} 1$ influenza viruses from swine in Jiangsu Province of China. Arch. Virol. 158, 2531-2541. http://dx.doi. org/10.1007/s00705-013-1771-y

Webster R, Hulse D (2005): Controlling avian flu at the source. Nature. 435, 415-416. http://dx.doi.org/10.1038/435415a

Webster RG, Bean WJ, Gorman OT, Chambers TM, Kawaoka Y (1992): Evolution and ecology of influenza A viruses. Microbiol. Mol. Biol. R. 56, 152-179.

Wu JM, Tu CC, Yu XL, Zhang ML, Zhang NZ, Zhao MY, Nie WX, Ren ZJ (2007): Bacteriophage T4 nanoparticle capsid surface SOC and HOC bipartite display with enhanced classical swine fever virus immunogenicity: a powerful immunological approach. J. Virol. Methods 139, 50-60. http://dx.doi.org/10.1016/j.jviromet.2006.09.017

Yao Q, Fischer KP, Li L, Agrawal B, Berhane Y, Tyrrell DL, Gutfreund KS, Pasick J (2010): Immunogenicity and protective efficacy of a DNA vaccine encoding a chimeric protein of avian influenza hemagglutinin subtype $\mathrm{H} 5$ fused to CD154 (CD40L) in Pekin ducks. Vaccine 28, 8147-8156. http://dx.doi.org/10.1016/j.vaccine.2010.09.081

Yu X, Jia RY, Huang J, Shu B, Zhu DK, Liu Q, Gao XH, Lin M, Yin ZQ, Wang MS, Chen S, Wang Y, Chen XY, Chen AC (2012): Attenuated Salmonella typhimurium delivering DNA vaccine encoding duck enteritis virus UL24 induced systemic and mucosal immune responses and conferred good protection against challenge. Vet. Res. 43,56 . http:// dx.doi.org/10.1186/1297-9716-43-56

Yun CH, Lillehoj HS, Choi KD (2000): Chicken IFN- $\gamma$ monoclonal antibodies and their application in enzyme-linked immunosorbent assay. Vet. Immunol. Immunop. 73, 297-308. http://dx.doi.org/10.1016/S0165-2427(00)00146-X 